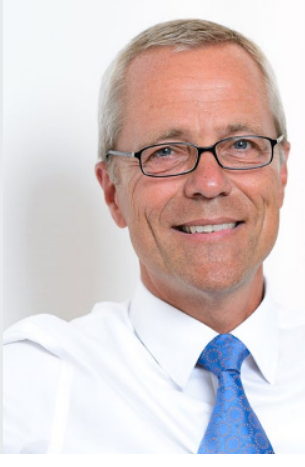
॥ Sorgen wir alle dafür, dass sich die DGNeurologie erfolgreich am
Markt etabliert!

Prof. Dr. G. R. Fink

Klinik und Poliklinik für Neurologie

Kerpener Straße 62, 50937 Köln, Deutschland

gereon.fink@uk-koeln.de

\title{
DGNeurologie ist die Zeitschrift von Mitgliedern der DGN für Mitglieder der DGN
}

Liebe Kolleginnen und Kollegen,

Sie halten nun die dritte Ausgabe unserer DGNeurologie in den Händen: Die „Geburt“ ist geglückt und es ist an der Zeit, allen Beteiligten, die dazu beitrugen, ein herzliches „Dankeschön“ zu sagen. Der Weg zur eigenen Zeitschrift, die der DGN (Deutsche Gesellschaft für Neurologie) gehört, war nicht einfach. Umso schöner ist es, zu sehen, dass es sich gelohnt hat! Was Sie in den Händen halten, ist eine moderne Zeitschrift, ganz anders gemacht als bisherige Zeitschriften, mit vielen innovativen Elementen - inhaltlich wie grafisch. Und v. a. eine Zeitschrift mit einem Herausgeberteam, das die Mitglieder der DGN in erfrischender Form widerspiegelt: Assistenzärzte, Oberärzte, Chefärzte, Ordinarien, Niedergelassene, Wissenschaftler - Sie alle sind vertreten, um das zu repräsentieren, was wir sind: Wir sind Neurologie! Und auch, dass die Neurologie zunehmend weiblich wird, lässt sich am Editorial Board ablesen.

Wir wurden oft gefragt: Warum eine neue Zeitschrift, eine weitere Zeitschrift? Warum eine Zeitschrift, die der Fachgesellschaft gehört? Viele von uns sind doch ohnehin als Autoren, Herausgeber, Gutachter usw. für schon mehr als genug Zeitschriften tätig! Genau darum ging es: Erstmals sind wir für uns selbst tätig: Wir, die Autoren, die Gutachter, die Herausgeber arbeiten für uns selbst, für die DGN! Gemacht wird eine Zeitschrift für die Mitglieder der DGN von Mitgliedern der DGN. Eine Zeitschrift, die Mitteilungen der Fachgesellschaft enthält, v. a. aber fachliche und wissenschaftliche Informationen mit hoher Alltagsrelevanz! „Herausgekommen“ ist eine Zeitschrift mit vielen innovativen Elementen: „standard operating procedures“ (SOP), Arzneimitteltherapie, Zoom! oder das Neuro-Quiz - alles mit Blick auf den Klinik- und Praxisalltag, bis hin zu aktuellen juristischen Fragen! Und so ist es nicht verwunderlich, dass die DGNeurologie Aufmerksamkeit auf sich zieht: nicht nur in der neurologischen Gemeinschaft, sondern auch bei Verlagen und anderen medizinischen Fachgesellschaften bis hin zu den Medien!

Es liegt in der Natur des Ansatzes, dass die DGNeurologie auch zu einer Änderung der deutschsprachigen neurologischen Zeitschriftenlandschaft führt. Sorgen wir gemeinsam dafür, dass unsere DGNeurologie sich erfolgreich am Markt der Zeitschriften etabliert und wir so eine ganz neue Erfolgsgeschichte in der Historie der DGN schreiben!
Ich bedanke mich bei allen, die zum Gelingen unserer DGNeurologie beitrugen, bei unserem Geschäftsführer, Herrn Dr. Thiekötter, der gemeinsam mit mir in zähen Verhandlungen mit unterschiedlichen Verlagen die rechtliche Grundlage für das schuf, was wir heute in den Händen halten. Ich bedanke mich bei dem Verlag (Springer), für den dies auch ein völlig neuer Weg ist, und bei allen Mitherausgebern, Autoren und Gutachtern. Es ist ihr Engagement, das zu einer modernen, unser Fach wirklich repräsentierenden Zeitschrift und einer lebendigen, erfolgreichen Fachgesellschaft beiträgt. Ganz besonders bedanke ich mich beim Schriftleiter, Herrn Prof. Dr. Berlit, der mit all seinem Know-how und seiner Erfahrung diese Zeitschrift gestaltet und so erfolgreich aufsetzte: Vielen, vielen Dank!

Ich wünsche Ihnen viel Spaß bei der Lektüre,

Ihr

Gereon Fink

Interessenkonflikt. G. R. Fink gibt an, dass kein Interessenkonflikt besteht. 